\title{
INFINITE GENERAL LINEAR GROUPS
}

\author{
BY \\ RICHARD V. KADISON( ${ }^{(1)}$
}

1. Introduction. In this paper, we carry out the investigation, promised in [4] $\left(^{2}\right)$, of the general linear group $\mathcal{X}_{g}$ of a factor $\mathcal{X}\left({ }^{3}\right)$, that is, the group of all invertible operators in $\mathcal{X}$. The results of this investigation have much in common with the results of [4]; and, where there is some basis for comparison, namely, in the case where $\mathcal{X}$ is a finite factor, there is a great deal of similarity between the present results and those of the classical finite-dimensional case. There is much greater resemblance between $\mathcal{X}_{g}$ and $\mathcal{N}_{g}$, with $\mathcal{X}$ a factor of type $\mathrm{II}_{1}$ and $\mathcal{N}$ a factor of type $\mathrm{I}_{n}$, than there is between the corresponding unitary groups, $\mathcal{X}_{u}$ and $\mathcal{N}_{u}$, of such factors. This circumstance results from the fact that the determinant function of [3] takes effect on $\mathscr{X}_{g}$ but not on $\mathscr{X}_{u}$ (cf. [3, Theorem 4]). The spectral theoretic and approximation techniques of [4] are stock methods in the present paper, and, for this reason, many of the proofs appearing here are reminiscent of those in [4]. There are, however, some striking differences in the methods required for the investigation of the general linear groups and those required for the investigation of the unitary groups. The most minor difference is the fact that the scalars involved do not form a compact set. This, in combination with the fact that the groups we handle are not locally compact, forces us, occasionally, to detour around what we have come to regard, through our experience with locally compact groups, as the most natural approach to the results obtained. In the second place, as we remarked above, the positive aspects of the determinant theory [3] come into play when studying the general linear group of a factor of type $\mathrm{II}_{1}$, whereas no positive aspect of the determinant theory is to be detected in the study of the unitary group of such a factor. Needless to say, the results of [3] must be used in the study of the general linear group of a factor of type $\mathrm{II}_{1}$. Finally, we must, in the present situation, deal with non-normal operators, since the general linear group of a factor consists primarily of such operators. This we deem to be the chief difficulty confronting the current investigation. In [4], of course, all the operators occurring were unitary and a fortiori normal. To be sure, there were difficulties, which could not be dealt with by spectral theoretic techniques, arising from the need to handle, simultaneously, noncommuting unitary operators.

Presented to the Society, December 29, 1952; received by the editors November 5, 1952.

(1) This work was done, in part, under contract with the O.N.R.

${ }^{2}$ ) Numbers in brackets refer to the bibliography at the end of this paper.

(3) For the general theory of factors which we shall use, refer to [5]. We shall make free use of the results of this memoir, as well as of the results of [3] and [4]. 
The difficulties could be isolated however (cf. [4, Lemma 6]), and managed by an approximation to the finite-dimensional case. The problems concerning non-normal operators, as they arise in the present case, require us to treat such operators as if they had a spectral decomposition, or, in analogy with the finite-dimensional case, as if they had a Jordan canonical form. Present day operator theory is not at the stage which allows one to come to grips with the non-normal operator in this powerful a manner.

The opening lemma of $\$ 3$ provides a means for circumventing the nonnormal operator difficulty in the $\mathrm{II}_{1}$ and III cases. This technique is only partially effective in the $\mathrm{I}_{\infty}$ and $\mathrm{II}_{\infty}$ cases. The problem, after this lemma, is left at the stage where the non-normal operators occurring satisfy an "approximate normality" condition (cf. Lemma 7). From this point the solution is completed, with great effort, by means of a direct attack using an "approximate spectral theory," but only in the $\mathrm{I}_{\infty}$ case (cf. Theorem 4 ).

In $\S 2$ we list some preliminary results which are needed later. $\S 3$ contains the main results. In this section, it is proved that each uniformly closed, noncentral, normal subgroup $G$ of the general linear group $\mathcal{X}_{g}$ of a factor $\mathscr{X}$ of type $\mathrm{II}_{1}$ is the inverse image under the determinant map (cf. [3]) of some closed subgroup of the positive reals, and also that the subgroup of $\mathcal{X l}_{g}$ consisting of those operators of determinant 1 is topologically simple (no proper, closed, noncentral, normal subgroups). If $\mathcal{X}$ is of type III then each such subgroup $G$ coincides with $\mathcal{X}_{g}$, and if $\mathcal{X}$ is of type $\mathrm{I}_{\infty}$ or $\mathrm{II}_{\infty}$, then $G$ contains $\mathscr{X}_{\theta f(1)}$ the closed, normal subgroup of $\mathscr{X}_{g}$ consisting of those operators the constituents of whose polar decomposition each has 1 as its unique center of infinite density (cf. [4, Definition 1, p. 393]). Each normal operator in $G$ is shown to lie in $\mathcal{X}_{g_{f}}$, the direct product of the nonzero scalars with $\mathscr{X}_{g_{f}(1)}$. The group $\mathcal{X}_{g_{f(1)}}$ is proved to be topologically simple (though clearly not algebraically simple). $\S 4$ contains an approximation result for invertible operators, completely analogous to Corollary 1 of [4], together with some comments concerning the results and proofs of the preceding section and possible extensions.

As in [4], we continue the conveniently ambiguous practice of using the same symbol for an orthogonal projection operator and its range, and speaking of these objects as if they were the same. Some of the arguments are long and rather involved. We preferred to keep them this way rather than split them into short lemmas with the consequent loss of perspective. As a consequence of this, however, and in order to keep the subscript-superscript notation from becoming too cumbersome, we are forced to reuse, in other portions of a proof, symbols which are used to designate auxiliary entities appearing only in one portion of the proof. We hope that this warning will serve to eliminate any difficulty which might otherwise arise from this ambiguity of notation. As for our general notation, small Greek letters are used to denote scalars. The letters $E, F, P, Q, R$ with subscripts and primes 
denote projection operators; $U, V, W$ denote unitary and partially isometric operators; $H, K$ denote positive operators; and $A, B, C, T$ are used for arbitrary operators. Capital script letters refer to sets of operators. The symbols $\Delta(A), D(E)$ refer to the determinant of $A$ (cf. [3]) and the relative dimension of the projection $E$ (cf. [5]), respectively.

2. Preliminaries. In this section, we collect several facts which will be needed in the succeeding sections. The next lemma gives a precise formula for the determinant of a simple type of operator in a factor of type $\mathrm{II}_{1}$. This formula is used in the study of the general linear group of such a factor.

LEMмA 1. If $\mathcal{X}$ is a factor of type $\mathrm{II}_{1}$ and $A$ is an operator in $\mathcal{X}$ of the form $\lambda_{1} E_{1}+\cdots+\lambda_{n} E_{n}$, where $E_{1}, \cdots, E_{n}$ are mutually orthogonal projections in XC, then

$$
\Delta(A)=\prod_{i=1}^{n}\left|\lambda_{i}\right|^{D\left(E_{i}\right)} .
$$

Proof. According to $[3$, p. 521], we have

$$
\begin{aligned}
\Delta(A) & =\exp T\left(\log \left[\left(A^{*} A\right)^{1 / 2}\right]\right)=\exp T\left(\log \left[\left|\lambda_{1}\right| E_{1}+\cdots+\left|\lambda_{n}\right| E_{n}\right]\right) \\
& =\exp \left[\left(\log \left|\lambda_{1}\right|\right) D\left(E_{1}\right)+\cdots+\left(\log \left|\lambda_{n}\right|\right) D\left(E_{n}\right)\right]=\prod_{i=1}^{n}\left|\lambda_{i}\right|^{D\left(E_{i}\right)} .
\end{aligned}
$$

We recall, next, several results from the finite-dimensional theory. If $\mathscr{X}$ is a factor of type $\mathrm{I}_{n}, n$ finite, and $\mathscr{X}_{0_{1}}$ is the subgroup of its general linear group $\mathscr{X}_{g}$ consisting of those elements in $\mathscr{X}_{g}$ which have determinant 1 (determinant understood, for the moment, in the standard (non-normalized) finite-dimensional sense), then each closed normal subgroup of $\mathcal{X}_{g_{1}}$ is contained in the center of $\mathscr{X}_{g_{1}}$ (those scalars which lie in $\mathscr{X}_{\sigma_{1}}$ ). Suppose $G$ is a closed, noncentral, normal subgroup of $\mathcal{X}_{g}$. The first portion of the proof of Lemma 4 is valid in this situation and implies that $G$ contains a noncentral, normal operator $A$. With $U$ a unitary operator in $\mathcal{X}, U^{-1} A^{-1} U A$ is in $\mathcal{X}_{g_{1}}$, and it is easy to see, in this case, that, for some unitary operator $U$, $U^{-1} A^{-1} U A$ is noncentral. Thus $\mathcal{X}_{g_{1}}$ intersects $G$ in a closed, noncentral normal subgroup of $\mathcal{X}_{g_{1}}$ and, hence, all of $\mathcal{X}_{g_{1}}$. We can state:

LEMMA 2. If $\mathcal{X}$ is a factor of type $\mathrm{I}_{n}$ and $G$ is a closed, noncentral, normal subgroup of $\mathcal{X}_{g}$, the general linear group of $\mathcal{X}$, then $G$ contains $\mathcal{X}_{g_{1}}$, the determinant 1 subgroup of $\mathcal{X}_{g}$. Moreover, if $G$ is closed, normal, and noncentral in $\mathcal{X}_{o_{1}}$, then $G$ coincides with $\mathcal{X}_{o_{1}}$.

It will be convenient to have some terminology which distinguishes between the standard (non-normalized) determinant of an operator relative to a $\mathrm{I}_{n}$ in which it lies and the determinant of this same operator relative to a $\mathrm{II}_{1}$ containing the $\mathrm{I}_{n}$. An operator $A$ in any factor $\mathcal{X}$ is said to have "complex matrix determinant 1 " when there exists, in $\mathcal{X}$, a finite set of orthogonal 
equivalent projections $P_{1}, \cdots, P_{k}$ and partial isometries $V_{i j}$ between these projections which, together with the projections, form a finite set of matrix units, $P A$ is in the algebra generated by these matrix units and has standard determinant 1 relative to this algebra while $(I-P) A$ is $I-P$, where $P$ is the sum of the $P_{i}$. With this terminology, we state:

Lemma 3. If $G$ is a closed, normal subgroup of $\mathcal{X}_{g}$, the general linear group of a factor $\mathcal{X}$, or of $\mathcal{X}_{g_{1}}$, the group of determinant 1 operators in $\mathcal{X}_{o}$ when $\mathcal{X}$ is of type $\mathrm{II}_{1}$, and

$$
A=\gamma_{1} P_{1}+\cdots+\gamma_{k} P_{k}+I-P
$$

has complex matrix determinant 1 relative to $P_{1}, \cdots, P_{k}$ and a fixed set of partial isometries $V_{i j}$ between them, $A$ lies in $G$, and $P A$ is noncentral in the algebra generated by the $P_{i}$ 's and the associated partial isometries, then $G$ contains all operators in the set $\mathcal{L}$ of operators which have complex matrix determinant 1 relative to the $P_{i}$ 's and the $V_{i j}$ 's.

Proof. The group $\mathcal{L}$ is isomorphic to the determinant 1 subgroup of some factor of type $\mathrm{I}_{k}$. Moreover, if $\mathcal{X}$ is of type $\mathrm{II}_{1}, \mathcal{L}$ is contained in $\mathcal{X}_{g_{1}}$. Indeed, each operator $B$ in $\mathcal{L}$ is the product of a unitary operator $U$ in $\mathcal{L}$ and a positive operator $H$ in $\mathcal{L}$. Now $U$ is in $\mathscr{X}_{g_{1}}$ as are all unitary operators in $\mathscr{X}$, and $H$ is a unitary transform of a positive operator $C$ in $\mathcal{L}$, where

$$
C=\alpha_{1} P_{1}+\cdots+\alpha_{k} P_{k}+I-P .
$$

According to Lemma 1,

$$
\Delta(C)=\prod_{i=1}^{k} \alpha_{i}^{D\left(P_{i}\right)}=\left[\prod_{i=1}^{k} \alpha_{i}\right]^{D\left(P_{1}\right)}=1 .
$$

Thus $\Delta(H)$ is 1 , and $B$ is in $\mathscr{X}_{\sigma_{1}}$. It follows that, in any case, the group $G$ intersects $\mathcal{L}$ in a closed, noncentral, normal subgroup of $\mathcal{L}$, and so, by Lemma 2, contains $\mathcal{L}$.

3. Main results. In this section, we list the information obtained concerning the closed normal subgroups of the general linear group of a factor. The first result of this section, Lemma 4, allows us to bring into play the results of [4] concerning the unitary group $\mathcal{X}_{u}$ of a factor $\mathcal{X}$ when dealing with the normal subgroups of its general linear group $\mathcal{X}_{g}$. Portions of the proof of this lemma, with slight alterations, are used at other points in this section.

Lemma 4 gives us sufficient information for dealing with the general linear group of a factor of type $\mathrm{II}_{1}$, and the classification of the closed, normal subgroups of this group is contained in Theorem 1, immediately following Lemma 4. However, Lemma 4 alone is not sufficient for handling the general linear groups of the infinite factors. A portion of the argument of Lemma 4 is incorporated in the proof of Lemma 5. This lemma is designed explicitly for the infinite cases. As in the unitary case, a normal subgroup of the general 
linear group of a factor is obtained by considering those operators whose nontrivial action is restricted to a subspace of finite relative dimension. The operators in the uniform closure of this subgroup are completely described in Theorem 2, and the relation of this subgroup to the general linear group of the various factors is given there. On the way to describing the closed, normal subgroups of the general linear groups of the factors, Lemma 6 contains a proof of the fact that each noncentral such subgroup contains the group $\mathscr{X}_{g_{f}(1)}$, consisting of operators in the uniform closure of the group of operators which act as the identity on the complement of a finite-dimensional subspace. The closed normal subgroups of a factor of type III are then described. Lemma 7 establishes the "approximate" normality of operators in a proper, closed, normal subgroup of the general linear groups of an infinite factor. With the aid of this lemma, the foregoing results, and some of the geometry of infinite-dimensional subspaces of a Hilbert space contained in [1], the closed normal subgroups of the general linear group of a factor of type $I_{\infty}$ are classified.

Lemma 4. If $\mathcal{X}$ is a factor, $\mathcal{X}_{g}$ its general linear group, and $G$ is a closed normal subgroup of $\mathcal{X}_{0}$ not contained in the center of $\mathcal{X}$, then $\mathcal{G}$ contains a noncentral unitary operator. If $\mathcal{X}$ is of type $\mathrm{II}_{1}$ or III, then $G$ contains $\mathcal{X}_{u}$, the full group of unitary operators of $\mathcal{X}$.

Proof. We first show that $G$ contains a noncentral normal operator. Suppose that $U H$ is the polar decomposition of some nonscalar in $G$ (cf. [5, p. 143]). Then $H^{-1} U^{-1}$ and $H\left(U^{-1}(U H) U\right) H^{-1}$ are in $G$ so that

$$
A=\left(H^{-1} U^{-1}\right)\left(H^{2} U H^{-1}\right)=H^{-1} U^{-1} H^{2} U H^{-1}
$$

is in $G$. Now $A$ is clearly positive definite. If $A$ is not a scalar we are through; if $A=\alpha I$ then $H^{2} U=\alpha U H^{2}$. Taking the norm of both sides of this last equality, we have $\alpha=|\alpha|=1$, so that $H^{2}$ commutes with $U$ and hence with $U^{2}$. But

$$
H^{-1}\left(\left(U^{-1}(U H) U\right) U H\right) H=U^{2} H^{2}
$$

is in $G$ and is normal (since $U^{2}$ and hence $U^{* 2}$ commute with $H^{2}$ ). If $U^{2} H^{2}$ is a scalar, the positive operator $H^{2}$ is a scalar multiple of a unitary operator which can occur only if $U^{2}$ is $I$ or $-I$ and $H$ is a scalar. In this case $G$ contains $U H$, a scalar multiple of the unitary operator $U$, which is noncentral by assumption. In any event we have our contention that $G$ contains a noncentral normal operator. Let $A$ be this operator and suppose first that $A$ has only point spectrum. If possible, choose points $\alpha, \beta$ in the spectrum of $A$ so that $\alpha$ is distinct from $\beta$ and $-\beta$. If this is not possible, then $A=\beta E-\beta(I-E)$. Since $\mathcal{H}$ may be assumed to be not of type $\mathrm{I}_{1}, \mathrm{I}_{2}$, it is possible to choose equivalent nonzero projections $F_{1}, F_{2}$ in $E$ and $I-E$ respectively such that one of $F_{1}, F_{2}$ does not coincide with $E$ or $I-E$, respectively. Let $V^{\prime}$ be a par- 
tial isometry from $F_{1}$ to $F_{2}$, and let $V$ be the unitary operator which is $V^{\prime}$ on $F_{1}, V^{*}$ on $F_{2}$, and the identity on $I-F_{1}-F_{2}$. Then

$$
V^{-1} A V A^{-1}=-\left(F_{1}+F_{2}\right)+I-F_{1}-F_{2},
$$

a noncentral unitary operator, is in $G$. Suppose then that $\alpha, \beta$ can be chosen in the (point) spectrum of $A$ so that $\alpha$ is distinct from $\beta$ and $-\beta$. Let $E, F$ be the spectral projections for $A$ corresponding to $\alpha$ and $\beta$, respectively. Choose equivalent, nonzero projections $E_{1}, F_{1}$ in $E$ and $F$ respectively; let $V^{\prime}$ be a partial isometry from $E_{1}$ to $F_{1}$, and let $V$ be the unitary operator which is $V^{\prime}$ on $E_{1}, V^{*}$ on $F_{1}$, and the identity on $I-E_{1}-F_{1}$. Then

$$
V^{-1} A V A^{-1}=\alpha^{-1} \beta E_{1}+\alpha \beta^{-1} F_{1}+I-E_{1}-F_{1},
$$

which is an operator in $G$ with complex matrix determinant 1 . This operator is noncentral, for if $\alpha^{-1} \beta=\alpha \beta^{-1}$ then $\alpha$ is $\beta$ or $-\beta$ contrary to choice. Thus $G$ contains, in particular, $i E_{1}-i F_{1}+I-E_{1}-F_{1}$, by Lemma 3 , a noncentral unitary operator.

We may assume, therefore, that $A$ has some continuous spectrum so that, in particular, $A$ has an infinite number of distinct points in its spectrum. It follows that we can choose two points $\alpha, \beta$ in the spectrum of $A$ so that either $\alpha$ and $\beta$ are both centers of infinite density for $A$ (cf. [4, p. 393]) or both are not centers of infinite density for $A$ and so that $\alpha$ is distinct from $\beta$ and $-\beta$. If possible, select $\alpha$ and $\beta$, satisfying the above conditions, so that they are not centers of infinite density for $A$. If $\alpha, \beta$ are centers of infinite density for $A$, choose orthogonal infinite projections $P, Q$ such that $I-P-Q$ is infinite; if $\alpha, \beta$ are not centers of infinite density, choose $P$ and $Q$ to be nonzero, orthogonal, equivalent, finite projections. Our aim now is to approximate, uniformly to within $\epsilon$, a unitary transform of $\alpha \beta^{-1} P+\alpha^{-1} \beta Q+I$ $-P-Q$ and hence $\alpha \beta^{-1} P+\alpha^{-1} \beta Q+I-P-Q$, itself, by an operator in $G$. It will follow then that $\alpha \beta^{-1} P+\alpha^{-1} \beta Q+I-P-Q$ is in the closure of $G$ and hence in $G$. Now, whether $P$ and $Q$ are infinite or not, $\alpha \beta^{-1} P+\alpha^{-1} \beta Q+I-P$ $-Q$ has complex matrix determinant 1 and is not central in its complex matrix ring since $\alpha \beta^{-1} \neq \alpha^{-1} \beta$. It follows from this that $G$ contains the noncentral unitary operator $i P-i Q+I-P-Q$. It remains to perform the approximation. For this purpose, choose nonintersecting circles of radius less than $\epsilon$ about $\alpha$ and $\beta$, these circles being so chosen in case $\alpha, \beta$ are not centers of infinite density for $A$ that the corresponding spectral projections $E, F$ are finite. Choose nonzero, orthogonal, equivalent projections $E_{1}, F_{1}$ in $E$ and $F$ respectively, which commute with $A$ and such that $E_{1}$ has $n$ copies in $P$ and $F_{1}$ has $n$ copies in $Q$ for some positive integer $n$ (i.e., $P$ and $Q$ are each the sum of $n$ orthogonal projections each equivalent to $E_{1}$ and $F_{1}$ ). If $\alpha$ and $\beta$ are not centers of infinite density for $A$, the possibility of choosing $E_{1}, F_{1}$ as indicated is guaranteed by [4, Lemma 5]. If $\alpha, \beta$ are centers of infinite density, they were selected because it was not possible to choose them otherwise, 
and this can be the case only if $A$ has at most a finite number of points in its spectrum which are not centers of infinite density. In this case, take $n=1$ and choose $E_{1}, F_{1}$ as above so that $I-E_{1}-F_{1}$ is infinite (choose them inside spectral projections for disks about $\alpha, \beta$ which avoid some disk about another center of infinite density). Let $V^{\prime}$ be a partial isometry from $E_{1}$ to $F_{1}$, and $V$ be the unitary operator which is $V^{\prime}$ on $E_{1}, V^{\prime *}$ on $F_{1}$, and the identity on $I-E_{1}-F_{1}$. Then $B=V^{-1} A V A^{-1}$ is in $G$. Choose $2 n-2$ orthogonal projections $E_{2}, \cdots, E_{n} ; F_{2}, \cdots, F_{n}$ in $I-E_{1}-F_{1}$ each equivalent to $E_{1}$, let $V_{i}^{\prime}$ be a partial isometry from $E_{1}$ to $E_{i}, V_{i}^{\prime \prime}$ a partial isometry from $F_{1}$ to $F_{i}$ and let $V_{i}$ be the unitary operator which is $V_{i}^{\prime}$ on $E_{1}, V_{i}^{\prime *}$ on $E_{i}, V_{i}^{\prime \prime}$ on $F_{1}, V_{1}^{\prime \prime *}$ on $F_{i}$, and the identity on $I-E_{1}-E_{i}-F_{1}-F_{i}$ (let $\left.V_{1}=I\right)$. Then

$$
T=\prod_{i=1}^{n} V_{i}^{-1} B V_{i}
$$

is in $G$. Moreover,

$$
\left\|T-\left\{\beta \alpha^{-1}\left(\sum_{i=1}^{n} E_{i}\right)+\beta^{-1} \alpha\left(\sum_{i=1}^{n} F_{i}\right)+I-\sum_{i=1}^{n}\left(E_{i}+F_{i}\right)\right\}\right\|<\epsilon,
$$

by construction, and

$$
\beta \alpha^{-1}\left(\sum_{i=1}^{n} E_{i}\right)+\beta^{-1} \alpha\left(\sum_{i=1}^{n} F_{i}\right)+I-\sum_{i=1}^{n}\left(E_{i}+F_{i}\right)
$$

is equivalent to

$$
\beta \alpha^{-1} P+\beta^{-1} \alpha Q+I-P-Q
$$

for, if $\mathcal{X}$ is a finite factor, the fact that $\sum_{i=1}^{n} E_{i}$ and $\sum_{i=1}^{n} F_{i}$ are equivalent to $P$ and $Q$ is sufficient to establish the equivalence; if $\mathcal{X}$ is infinite and $E_{1}, F_{1}$ are finite then $I-\sum_{i=1}^{n}\left(E_{i}+F_{i}\right)$ is infinite as is $I-P-Q$, by choice, and, finally, if $E_{1}, F_{1}$ are infinite then $n=1$, and $I-\sum_{i=1}^{n}\left(E_{i}+F_{i}\right)=I-E_{1}$ $-F_{1}$ which is infinite, by construction, as is $I-P-Q$. Thus, in all cases, we have performed the desired approximation, and the proof is complete. Of course, the last statement of this lemma follows from the information we already have and the results concerning $\mathcal{X}_{u}$ for $\mathcal{X}$ a factor of type $\mathrm{II}_{1}$ or III [4, Theorem 2].

Making use of the above lemma and the determinant theory of [3], we are able to completely classify the closed, normal subgroups of the general linear group of a factor of type $\mathrm{II}_{1}$. This classification is contained in the following theorem.

THEOREM 1. If $\mathcal{X}$ is a finite factor and $\Delta$ is the determinant function on $\mathcal{X}$, then all uniformly closed, noncentral, normal subgroups of $\mathcal{X}_{g}$, the general linear group of $\mathcal{X}$, appear as the inverse images under $\Delta$ of closed subgroups of the range of $\Delta$. Moreover, $\mathcal{X}_{g_{1}}$, the subgroup of $\mathcal{X}_{0}$ consisting of those elements in $\mathcal{X}_{g}$, with 
determinant 1 , has as its closed normal subgroups the closed central (scalar) subgroups.

Proof. Suppose we can show that each closed, noncentral, normal subgroup $\mathcal{G}$ of $\mathscr{X}_{g}$ contains $\mathscr{X}_{g_{1}}$. Then, since $\mathscr{X}_{g_{1}}$ is the kernel of $\Delta$ in $\mathscr{X}_{g}, \Delta$ maps $\mathcal{X}_{\sigma} / \mathcal{X}_{g_{1}}$ continuously, isomorphically onto the group of positive reals, so that $G$ maps into some subgroup $\Delta(G)$ of the positive reals. As soon as we show that the map from $\mathcal{X}_{g} / \mathcal{X}_{g_{1}}$ to the positive reals is a topological isomorphism, we shall know that $\Delta(G)$ is closed. Now a neighborhood of the identity in $\mathcal{X}_{g} / \mathcal{X}_{g_{1}}$ can be represented as $\mathcal{U} \mathcal{X}_{g_{1}}$, with $\mathcal{U}$ a neighborhood of $I$ in $\mathcal{X}_{g}$; and $U$ intersects the set of scalars in an open set $\mathcal{S}$. Thus, since $\Delta(\alpha I)$ is $\alpha$, for $\alpha$ positive, the image of $U \cap\{\gamma I\}$ is an open subset of the positive reals. Thus the closed noncentral, normal subgroups of $\mathcal{X}_{g}$ (which, we have assumed, automatically contain $\mathcal{X}_{g_{1}}$ ) correspond, in 1-1 fashion, to the closed subgroups of the positive reals.

It remains to prove that a closed normal subgroup of $\mathcal{X}_{g}$ or $\mathcal{X}_{g_{1}}$ which is noncentral contains $\mathcal{X}_{g_{1}}$. Let $G$ be such a subgroup. We shall prove this by showing that $G$ contains all operators $A$ of the form

$$
A=\alpha_{1} E_{1}+\cdots+\alpha_{n} E_{n}
$$

with $\alpha_{1}, \cdots, \alpha_{n}$ positive, the $E_{i}$ mutually orthogonal projections with sum $I$ and

$$
\Delta(A)=\prod_{i=1}^{n} \alpha_{i}{ }^{D\left(E_{i}\right)}=1 .
$$

We then observe that these operators lie dense in the set of all positive operators in $\mathcal{X}_{g_{1}}$. If $G$ is a closed, noncentral, normal subgroup of $\mathcal{X}_{g_{1}}$, the proof of Lemma 4 remains valid for $G$, so that, in any case, $G$ contains $\mathcal{X}_{u}$, the unitary group of $\mathcal{X}$. Since $G$ contains $\mathcal{X}_{u}$ and all positive operators in $\mathscr{X}_{\sigma_{1}}, G$ contains $\mathscr{X}_{\sigma_{1}}$, for $G$ contains the constituent parts of the polar decomposition of each operator in $\mathcal{X}_{\boldsymbol{g}_{1}}$.

Suppose we have shown that $G$ contains all operators having the same general form as $A$ but involving $n-1$ or fewer projections $(n \geqq 3)$. Write

$$
B=\alpha_{1} E_{1}+\cdots+\alpha_{n-2} E_{n-2}+\alpha\left(E_{n-1}+E_{n}\right),
$$

where $\alpha$ is so chosen that $\Delta(B)$ is 1 . By inductive assumption, $B$ is in $G$ and

$$
B^{-1} A=\alpha^{-1} \alpha_{n-1} E_{n-1}+\alpha^{-1} \alpha_{n} E_{n}+I-E_{n-1}-E_{n}
$$

has determinant 1 . It suffices, therefore, to prove that $G$ contains all operators $C$ of the form

$$
C=\alpha E+\beta F+I-E-F,
$$

with $E, F$ orthogonal projections and 


$$
\Delta(C)=\alpha^{D(E)} \beta^{D(F)}=1,
$$

in order to show that $G$ contains all operators having the same form as $A$. We may deal with the case where $I-E-F$ is different from 0 , for, if we have the result in this case and $\alpha E+\beta F$ is given with

$$
E+F=I, \quad \alpha^{D(E)} \beta^{D(F)}=1 .
$$

Choose $P_{1}, P_{2}$ orthogonal, equivalent projections with sum $E$ and $Q_{1}, Q_{2}$ orthogonal, equivalent projections with sum $F$. By assumption, $\alpha P_{1}+\beta Q_{1}$ $+I-P_{1}-Q_{1}$ and $\alpha P_{2}+\beta Q_{2}+I-P_{2}-Q_{2}$ are in $G$, so that their product $\alpha E+\beta F$ is in $G$. We show now that $C$, with $I-E-F$ nonzero, can be approximated uniformly to within any preassigned positive $\epsilon$ by an operator in $G$. Observe first that $G$ contains all operators of the form $\gamma P+\delta Q+I$ $-P-Q$ with

$$
\gamma^{D(P)} \delta^{D(Q)}=1, \quad D(P) / D(Q)=m / n,
$$

where $m / n$ is some rational number. Indeed, decomposing $P$ into $m$ orthogonal equivalent projections and $Q$ into $n$ orthogonal equivalent projections yields $m+n$ orthogonal equivalent projections, and $\gamma P+\delta Q+I-P-Q$, viewed in this decomposition, has complex matrix determinant

$$
\gamma^{m} \delta^{n}=\left(\gamma^{m / n} \delta\right)^{n}=\left(\gamma^{D(P)} \delta^{D(Q)}\right)^{n / D(Q)}=1 .
$$

Since $G$ contains the unitary operators in $\mathcal{X}, G$ contains $\gamma P+\delta Q+I-P-Q$, by Lemma 3. In the case of $C$, therefore, we may assume that $D(E) / D(F)$ is not rational. Choose an integer $n$ so large and a projection $E_{1}$ in $E$ with $D\left(E_{1}\right)$ so close to $D(E)$ that the following conditions are fulfilled: $D\left(E_{1}\right) / D(F)$ is a rational number,

$$
\left|\alpha^{-1 / n}-1\right|<\epsilon, \quad n D\left(E-E_{1}\right) \leqq D(I-E-F), \quad\left|\alpha_{1}-\alpha\right|<\epsilon,
$$

where $\alpha_{1}$ is $\beta^{-D(F) / D\left(E_{1}\right)}$ (note that $\alpha$ is $\beta^{-D(F) / D(E)}$ ). With these choices made, let $P_{1}, \cdots, P_{n}$ be $n$ orthogonal equivalent projections in $I-E-F$, each having the same dimension as $E-E_{1}$. Then, by our preceding remarks, $\alpha_{1} E_{1}+\beta F+I-E_{1}-F$ is in $G$, since $D\left(E_{1}\right) / D(F)$ is rational and

$$
\alpha_{1}^{D\left(E_{1}\right)} \beta^{D(F)}=1 \text {. }
$$

Moreover,

$$
\alpha\left(E-E_{1}\right)+\alpha^{-1 / n}\left(P_{1}+\cdots+P_{n}\right)+I-\left(E-E_{1}\right)-P_{1}-\cdots-P_{n}
$$

is in $G$, since it has complex matrix determinant 1 ; so that the product

$$
\begin{aligned}
T= & \alpha\left(E-E_{1}\right)+\alpha_{1} E_{1}+\beta F+\alpha^{-1 / n}\left(P_{1}+\cdots+P_{n}\right) \\
& +I-E-F-P_{1}-\cdots-P_{n}
\end{aligned}
$$

of these two operators is in $G$. But 


$$
\|C-T\|<\epsilon,
$$

and we conclude that $G$ contains all operators having the form of $A$.

If a positive $H$ in $\mathcal{X}_{\theta_{1}}$ is given choose

$$
K=\alpha_{1} E_{1}+\cdots+\alpha_{n} E_{n}
$$

with $\alpha_{1}, \cdots, \alpha_{n}$ positive, $E_{1}, \cdots, E_{n}$ orthogonal projections,

$$
\|H-K\|<\delta / 2
$$

where $\delta$ is a small, preassigned, positive number, and

$$
\left|1-(\Delta(K))^{-1}\right|<\delta / 4\|H\| \text {. }
$$

The choice of such a $K$ is made possible by the spectral theorem and the uniform continuity of $\Delta$ on $G$ (cf. [3, Theorem 1, ( $\left.\left.3^{\circ}\right)\right]$ ). Then

$$
\Delta(K / \Delta(K))=1,
$$

and

$$
\begin{aligned}
\|H-K / \Delta(K)\| & \leqq\|H-K\|+\|K-K / \Delta(K)\| \leqq \delta / 2+\|K\| \cdot\left|1-(\Delta(K))^{-1}\right| \\
& \leqq \delta / 2+2\|H\| \delta / 4\|H\|=\delta .
\end{aligned}
$$

How $K / \Delta(K)$ is in $G$ so that $H$ is in $G$ and the proof is complete.

For the remainder of this section, we are concerned with the general linear groups of the various infinite factors. The next lemma provides a means of determining restrictions on the structure of an operator which can lie in a closed, proper, normal, subgroup of the general linear group of an infinite factor.

Lemma 5. If $\mathcal{X}$ is an infinite factor, $\mathcal{X}_{o}$ its general linear group, $G$ a closed, normal subgroup of $\mathcal{X}_{0}$ containing the noncentral operator $A=\alpha_{1} E_{1}+\ldots$ $+\alpha_{n} E_{n}$, where $E_{1}, \cdots, E_{n}$ are infinite orthogonal projections in $\mathcal{X}$, then $G$ is identical with $\mathcal{X}_{g}$.

Proof. The invertibility of $A$ and the orthogonality of the $E_{i}$ imply that $I=E_{1}+\cdots+E_{n}$. The fact that $A$ is noncentral implies that $n>1$ and not all the $\alpha_{i}$ are equal.

With the following small adjustments, the first portion of the proof of Lemma 4 shows that our $G$ contains $\mathcal{H}_{u}$. We have the noncentral normal operator $A$ given us in the present situation, and it has pure point spectrum consisting only of centers of infinite density. In the first case of the proof of Lemma 4, when $A$ has only the points $\beta,-\beta$ in its spectrum, we choose the $F_{1}, F_{2}$ of this argument infinite such that $I-F_{1}-F_{2}$ is infinite, as can be done in the present situation. The noncentral unitary, $-\left(F_{1}+F_{2}\right)+I-F_{1}$ $-F_{2}$, has the two centers of infinite density $1,-1$ and lies in $G$, as shown. In the other case of the proof of Lemma 4 , where $\alpha, \beta$ can be chosen in the 
point spectrum of $A$ so that $\alpha$ is distinct from $\beta$ and $-\beta$, choose the $E_{1}, F_{1}$ of this argument infinite with $I-E_{1}-F_{1}$ infinite. The noncentral unitary operator constructed in this case, $i E_{1}-i F_{1}+I-E_{1}-F_{1}$, has $i,-i$ as centers of infinite density and lies in $G$. Thus, in any event, the $G$ of the present situation contains a unitary operator not in $G_{f}$, the group of unitary operators in $\mathcal{X}$ which have exactly one center of infinite density so that $G$ contains $\mathfrak{X}_{u}$, by $[4$, Theorem 4$]$.

Our remaining task is to show that $G$ contains all positive invertible operators in $\mathcal{X}$ whence the lemma will follow from the fact that each operator in $G$ has a polar decomposition as the product of a unitary and a positive invertible operator. It will be sufficient to show that $G$ contains all operators of the form $\beta_{1} F_{1}+\cdots+\beta_{m} F_{m}$ with $F_{1}, \cdots, F_{m}$ orthogonal projections with sum $I$ and $\beta_{1}, \cdots, \beta_{m}$ positive numbers, by the spectral theorem and the uniform closedness of $G$, and, to show this, it suffices to show that $G$ contains all operators of the form $\beta F+I-F$ with $F$ an arbitrary projection in $\mathcal{H}$ and $\beta$ positive.

Suppose, first that $I-F$ is infinite. Let a positive $\epsilon$ be given and choose $n$ so large that

$$
\left|1-\beta^{-1 / n}\right|<\epsilon .
$$

Let $F_{1}, \cdots, F_{n}$ be $n$ orthogonal projections contained in $I-F$ each equivalent to $F$. Since $G$ contains the unitary operators in $\mathcal{H}$, and

$$
B=\beta F+\beta^{-1 / n}\left(F_{1}+\cdots+F_{n}\right)+I-F-F_{1}-\cdots-F_{n}
$$

has complex matrix determinant $1, B$ lies in $G$, by Lemma 3. But

$$
\|\beta F+I-F-B\|<\epsilon
$$

so that $\beta F+I-F$ is in $G$. Choose $E$ so that $E$ and $I-E$ are infinite. Then $\beta E+I-E$ and $E+\beta(I-E)$ are in $G$, by the foregoing, so that their product $\beta I$ is in $G$. If now $I-F$, above, is finite, then $\beta I$ and $F+\beta^{-1}(I-F)$ are in $G$, so that their product $\beta F+I-F$ is in $G$; and the proof is complete.

As in the case of the unitary group, $\mathcal{X}_{u}$, of a factor $\mathcal{X}$, we should expect that those operators in $\mathcal{X}_{g}$ which are scalars on the complement of some subspace of finite relative dimension (denote this set by $\mathcal{X}_{g^{\prime} f}$ ) forms a normal subgroup of $\mathscr{X}_{g}$. It is easy to see that this is the case (cf. [4, Theorem 3]). The group $\mathcal{X}_{g^{\prime},}$ is, of course, not uniformly closed (in the $\mathrm{I}_{\infty}, \mathrm{II}_{\infty}$ cases). In [4] we were able to identify the closure of the corresponding group, $G_{f}^{\prime}$, in $\mathcal{X}_{u}$ as the set of those operators in $\mathcal{H}_{u}$ which have at most one center of infinite density in their spectrum (cf. [4, Definition 1]). In order to define "center of infinite density" for a normal operator, it was necessary to make use of the spectral decomposition of the operator. Of course, no such procedure is available to us in the case of operators in $\mathcal{X}_{g}$, since they are, for the 
most part, non-normal. In this situation, however, the polar decomposition works very nicely to our advantage, and we can state:

TheOREM 2. The uniform closure, $\mathcal{X}_{g_{f}}$, of $\mathcal{X}_{g^{\prime} f}$ in $M_{\theta}$, the general linear group of a factor $\mathcal{X}$, is a closed normal subgroup of $\mathcal{X}_{g}$ and consists of those operators in $\mathcal{X}_{0}$ the constituents of whose polar decomposition each have at most one center of infinite density. In the case of a finite factor $\mathcal{X}, \mathcal{X}_{g_{f}}=\mathcal{X X}_{g}$; if $\mathcal{X}$ is of type III, $\mathcal{X}_{\theta_{f}}=\{\lambda I\}$; if $\mathcal{X}$ is of type $\mathrm{I}_{\infty}$ or $\mathrm{II}_{\infty}, \mathcal{X}_{\theta_{f}}$ is proper and noncentral.

Proof. Suppose $A=U H, U$ unitary, $H$ positive invertible, is the polar decomposition of an operator $A$ in $\mathscr{X}_{g}$; and suppose $U$ has $\zeta$ as its only center of infinite density, $H$ has $\alpha$ as its only center of infinite density. Then

$$
\left\|U-\left(\zeta E_{\epsilon}+\left(I-E_{\epsilon}\right) U\right)\right\|<\epsilon, \quad \| H-\left(\alpha F_{\epsilon}+\left(I-F_{\epsilon}\right) H \|<\epsilon,\right.
$$

where $E_{\epsilon}$ and $F_{\epsilon}$ are spectral projections for $U$ and $H$, respectively, corresponding to open disks of radius less than $\epsilon$ about $\zeta, \alpha$, respectively. Now $\left[\alpha F_{\epsilon}+\left(I-F_{\epsilon}\right) H\right]\left[\zeta E_{\epsilon}+\left(I-E_{\epsilon}\right) U\right]$ is in $\mathscr{X}_{\theta^{\prime} f}$, so that $U H$ is a uniform limit point of $\mathscr{X}_{\sigma^{\prime} f}$ and, therefore, in $\mathscr{X}_{g f}$.

Let $V K, V$ unitary, $K$ positive, invertible, be the polar decomposition of an operator $B$ in $\mathscr{X}_{g_{f}}$, and suppose that $B_{n}$ in $\mathscr{X}_{g^{\prime},}$ tends uniformly to $B$. If $V_{n} K_{n}$ is the polar decomposition of $B_{n}$, then $K_{n}\left(=\left(B_{n}^{*} B_{n}\right)^{1 / 2}\right)$ tends uniformly to $K\left(=\left(B^{*} B\right)^{1 / 2}\right)$ and $V_{n}\left(=B_{n} K_{n}^{-1}\right)$ tends uniformly to $V\left(=B K^{-1}\right)$. Now $V_{n}$ and $K_{n}$ are in $\mathcal{X}_{g^{\prime} f}$, so that $V$ and $K$ are in $\mathcal{X}_{g f}$. But $V$ and $K$ are normal operators, and, for normal operators, the last paragraph of the proof of [4, Theorem 3] may be carried over verbatim to show that $V$ and $K$ each have at most one center of infinite density.

If $\mathscr{X}$ is finite, it is immediate from the definitions that $\mathcal{X}_{g^{\prime} f}=\mathscr{X}_{g}$, and, if $\mathcal{X}$ is of type III, that $\mathcal{X}_{g^{\prime} f}=\{\lambda I\}$, from which our assertion concerning these factors is immediate. If $\mathcal{X}$ is of type $\mathrm{I}_{\infty}$ or $\mathrm{II}_{\infty}, \mathscr{X}_{g_{f}}$ certainly contains more than the scalars. On the other hand, the unitary operator $E-(I-E)$, with $E$ and $I-E$ infinite, has the two distinct centers of infinite density 1 and -1 so that it is not in $\mathcal{X}_{o_{j}}$, which is, accordingly, proper.

We denote by $\mathscr{X}_{g f(1)}$ the normal subgroup of $\mathscr{X}_{g}$ consisting of the uniform closure of those operators which act as the identity on a subspace whose complement has finite relative dimension.

Lemma 6. If $\mathcal{X}$ is an infinite factor and $G$ is a uniformly closed, proper, noncentral, normal subgroup of $\mathcal{X}_{g}$, then $G$ contains $\mathcal{X}_{g_{f}(1)}, \mathcal{X}_{g_{f}(1)}$ is topologically simple, and each normal operator in $G$ lies in $\mathcal{X}_{\sigma_{f}}$.

Proof. According to Lemma $4, G$ contains a noncentral unitary operator and therefore, by [4, Theorems 2 and 4 ], all of $G_{f}^{(1)}$, the group of unitary operators which have 1 as their unique center of infinite density. Let a finite projection $E$ be given. The group of operators which act as the identity on $I-E$ and are invertible is topologically isomorphic to the general linear 
group of the finite factor $\mathscr{X}_{E}$ (cf. [5, pp. 186-188]). The intersection of $G$ with this group of operators in $\mathscr{X}_{\theta}$ is a closed, normal subgroup of this group and is noncentral (when imbedded in $\mathscr{X}_{E}$ ) unless $\mathcal{X}$ is type $\mathrm{I}_{\infty}$ and $E$ is 1-dimensional; for this intersection contains any operator which is unitary on $E$ and the identity on $I-E$. Thus, in any event, $G$ contains those operators which act as the identity on $I-E$ and have determinant 1 on $E$ (i.e., have determinant 1 when mapped into $\mathscr{X}_{E}$ ), by Theorem 1 . Let a positive scalar $\gamma$ be given and choose $n$ so large that $\left|1-\gamma^{-1 / n}\right|<\epsilon$, where $\epsilon$ is a preassigned positive number. Let $E_{1}, \cdots, E_{n}$ be $n$ orthogonal projections in $I-E$ each equivalent to $E$ (this choice is possible, of course, since $I-E$ is infinite). Now the operator

$$
B=\gamma E+\gamma^{-1 / n}\left(E_{1}+\cdots+E_{n}\right)+I-\left(E_{1}+\cdots+E_{n}\right)
$$

is in $G$, by the result immediately preceding, since it has determinant 1 on the finite projection $E+E_{1}+\cdots+E_{n}$. Moreover

$$
\|B-\gamma E-(I-E)\|<\epsilon .
$$

Since $G$ is uniformly closed, $\gamma E+I-E$ is in $G$, for each positive scalar, and, since $G$ contains the determinant 1 operators on $E, G$ contains all operators in $\mathcal{X}_{\theta}$ which act as the identity on $I-E$, by Theorem 1 . Since $E$ was an arbitrarily chosen, finite projection in $\mathscr{X}, G$ contains $\mathcal{X}_{g_{f}(1)}$.

In order to show that $\mathcal{X}_{g_{f}(1)}$ is topologically simple, we need only establish the fact that $\mathfrak{H}$, a closed, noncentral, normal subgroup of $\mathcal{X}_{g_{f(1)}}$, contains a noncentral, unitary operator. In fact, in [4, Theorem 4] we showed that $G_{f}^{(1)}$ is topologically simple; so that $\mathfrak{F C}$ would then contain all of $G_{f}^{(1)}$, and the above argument could be repeated verbatim for $\mathfrak{H C}$ to show that $\mathfrak{H C}$ contains $\mathcal{X}_{g_{f(1)}}$, and hence is identical with $\mathcal{X}_{g_{f(1)}}$. It remains to show that $\mathfrak{H C}$ contains a noncentral unitary operator if it is noncentral. We observe, first, that the argument of the first portion of Lemma 4 is valid for this situation and $\mathcal{H C}$ contains a noncentral, normal operator (for the operator $A$ of Lemma 4 has a polar decomposition, in the present case, whose constituents lie in $\mathcal{X}_{g_{f}(1)}$, according to Theorem 2, so that the inner transforms of $A$ required for the argument of that lemma all lie in $\mathcal{F}$ ). Let $A$ be this noncentral, normal operator in $\mathcal{H}$. If $A$ has at most three points in its spectrum, certainly one of these is 1 . Let the others be $\alpha, \beta$ and let $E$ be a projection in $\mathcal{X}$ which is the sum of the following three orthogonal projections: the spectral projections for $\alpha, \beta$, and a finite-dimensional subspace of the spectral projection corresponding to 1 . Now $A$ leaves $E$ invariant and is not a scalar on $E$. Thus, we can find some product of inner transforms of $A$ and its inverse as close to -1 on $E$ as we desire by Theorem 1 . Each element by which we take an inner transform of $A$ (or $A^{-1}$ ) is 1 on $I-E$ and hence in $\mathcal{X}_{g_{f}(1)}$, so that the resulting product is in $\mathcal{H}$. Since $\mathcal{H C}$ is closed, it contains $-E+I-E$ which is a noncentral unitary. We may suppose, therefore, that the spectrum of $A$ 
contains more than three points, so that it is possible to choose $\alpha$ and $\beta$ distinct from 1, in the spectrum of $A$, and such that $\alpha$ is distinct from $\beta$ and $-\beta$. From this point, it is possible to follow the last paragraph of the proof of Lemma 4 verbatim (simplifying it, indeed, by omitting those portions which refer to the case where $\alpha$ and $\beta$ are centers of infinite density for $A$, since they cannot be so in the present situation), and we conclude that $\mathfrak{F C}$ contains a noncentral unitary. Thus $\mathcal{X}_{g_{f(1)}}$ is topologically simple.

Suppose now that $A$ is a normal operator in $G$, a proper, closed, normal subgroup of $\mathcal{X}_{g}$. We wish to show that $A$ lies in $\mathcal{X}_{g f}$. Suppose that the spectrum of $A$ contains exactly two centers of infinite density $\alpha$ and $\beta$, and let $P$ be an infinite projection with $I-P$ infinite. Let $E$ and $F$ be orthogonal spectral projections for $A$ corresponding to disks of radius $\epsilon$ about $\alpha$ and $\beta$, respectively. Then $I-E-F$ is finite, since $\alpha$ and $\beta$ are the only centers of infinite density for $A$, so that $\beta A^{-1}(I-E-F)+E+F$ is in $\mathscr{X}_{g_{f}(1)}$ and hence in $G$. Thus

$$
A\left[\beta A^{-1}(I-E-F)+E+F\right]=A E+A F+\beta(I-E-F)
$$

is in $G$. But

$$
\|A E+A F+\beta(I-E-F)-[\alpha E+\beta(I-E)]\|<\epsilon,
$$

and $\alpha E+\beta(I-E)$ is a unitary transform of $\alpha P+\beta(I-P)$. Thus, since $G$ is uniformly closed, $\alpha P+\beta(I-P)$ is in $G$ which is all of $\mathcal{X}_{a}$, according to Lemma 5.

Suppose now that $A$ has three or more centers of infinite density. Let $\alpha$ and $\beta$ be two such centers for $A$, and let $P_{1}$ and $P_{2}$ be given infinite projections with $I-P_{1}-P_{2}$ infinite. Let a positive $\epsilon$ be given, and let $E^{\prime}, F^{\prime}$ be orthogonal spectral projections for $A$ corresponding to disks of radius less than $\epsilon$ about $\alpha$ and $\beta$, respectively, and such that $I-E^{\prime}-F^{\prime}$ is infinite (this is possible, since $A$ has at least three centers of infinite density). Let $V^{\prime}$ be a partial isometry from $E^{\prime}$ to $F^{\prime}$, and let $V$ be the unitary operator which is $V^{\prime}$ on $E^{\prime}$, $V^{\prime *}$ on $F^{\prime}$, and the identity on $I-E^{\prime}-F^{\prime}$. Then

$$
\left\|A^{-1} V^{*} A V-\left(\alpha^{-1} \beta E^{\prime}+\alpha \beta^{-1} F^{\prime}+I-E^{\prime}-F^{\prime}\right)\right\|<\epsilon
$$

and $\alpha^{-1} \beta E^{\prime}+\alpha \beta^{-1} F^{\prime}+I-E^{\prime}-F^{\prime}$ is a unitary transform of $\alpha^{-1} \beta P_{1}+\alpha \beta^{-1} P_{2}$ $+I-P_{1}-P_{2}$ which is accordingly in $G$, since $G$ is uniformly closed. The application of Lemma 5 completes the argument and the proof of the lemma.

There is little difficulty, now, in describing the closed, normal subgroups of the general linear group of a factor of type III. This is done in the following theorem.

TheOREM 3. If $\mathcal{X}$ is a factor of type III, then its general linear group $\mathfrak{X}_{0}$ contains no proper, uniformly closed, noncentral, normal subgroups.

Proof. Suppose $G$ is a closed, normal subgroup of $\mathcal{X}_{g}$. If $G$ is not contained in the center of $\mathcal{X}_{g}$ then $G$ contains $\mathscr{X}_{u}$, the unitary group of $\mathscr{X}$, according to 
Lemma 4. Thus, with $E$ and $I-E$ nonzero, the normal (unitary) operator $E-(I-E)$ lies in $G$ and has two centers of infinite density, 1 and -1 . Thus, by Lemma 6, $G$ coincides with $\mathcal{X}_{g}$.

Having obtained the information that each normal operator in a proper, closed, normal subgroup $G$ of $\mathscr{X}_{g}$, with $\mathscr{X}$ an infinite factor, lies in $\mathcal{X}_{g f}$, we proceed to establish this fact for the non-normal operators in $G$. Of course, in this case, the spectral theory is not directly available to us. We shall show, however, that the operators in $G$ have "large" portions which are "approximately normal," and then we apply the spectral theory in an approximate form.

The next lemma establishes the "approximate normality" of "large" portions of operators in $G$.

Lemma 7. If $G$ is a proper, closed, normal subgroup of $\mathcal{X}_{g}$, the general linear group of the infinite factor $\mathcal{X}$, and $U H, U$ unitary, $H$ positive, is the polar decomposition of an operator in $G$, then $U H U^{-1} H^{-1}$ and $U^{-1} H^{-1} U H$ are in $\mathcal{X}_{g_{f}(1)}$.

Proof. Suppose that both $A$ and $A^{*}$ are in $G$. Then $A^{*} A$ is in $G$, and, being self-adjoint, $A^{*} A$ is $\mathcal{X}_{g_{f}}$, by Lemma 6 . It follows that $\left(A^{*} A\right)^{1 / 2}$ is in $\mathcal{X}_{g_{f}}$, for if $A^{*} A$ is the uniform limit of $B_{n}$ with $B_{n}$ in $\mathcal{X}_{g^{\prime} f}$, then $A^{*} A$ is the uniform limit of $\left(B_{n}+B_{n}^{*}\right) / 2$ which is in $\mathcal{X}_{g^{\prime} f}$. We may assume, therefore, that $B_{n}$ is self-adjoint and, indeed, positive since $A^{*} A$ is positive, invertible, so that $B_{n}$ is positive for all but a finite number of $n$. It is clear that $B_{n}^{1 / 2}$ is in $\mathcal{X}_{g^{\prime} f}$, and $\left(A^{*} A\right)^{1 / 2}$ is the uniform limit of $B_{n}^{1 / 2}$. Thus some nonzero scalar multiple of $\left(A^{*} A\right)^{1 / 2}$ lies in $\mathcal{X}_{g f(1)}$ and hence in $G$. If we express $A$ in its polar decomposition $U H$ with $H=\left(A^{*} A\right)^{1 / 2}$ and $U$ unitary it follows that $U\left(=A\left(A^{*} A\right)^{-1 / 2}\right)$ has a scalar multiple which lies in $G$. This scalar multiple is certainly normal, so that, by our preceding results, it lies in $\mathcal{X}_{g_{f}}$. Now suppose $A$ in $G$ is expressible as the product of two self-adjoint invertible operators $H$ and $K$ (not necessarily in $G$ ). Then $A=H K$ is conjugate to its adjoint $A^{*}=K H=$ $=H^{-1}(H K) H$, so that $A^{*}$ is in $G$, and $A$ is in $\mathscr{X}_{g f}$, from the foregoing.

Suppose now that $A$ is an arbitrary operator in $G$ with polar decomposition $U H$. Then $A^{-1}=H^{-1} U^{-1}$ is in $G$ as is $U^{-1} H^{-1}=U^{-1}\left(H^{-1} U^{-1}\right) U$, so that ( $\left.U H U^{-1}\right) H^{-1}$ is in $G$. But $U H U^{-1}$ and $H^{-1}$ are positive, invertible, so that, by the above, $U H U^{-1} H^{-1}$ is in $\mathscr{X}_{g f}$. We shall show now that $U H U^{-1} H^{-1}$ is actually in $\mathscr{X}_{g_{(1)}(1)}$. Indeed, let $U H U^{-1} H^{-1}$ be the uniform limit if $B_{n}$ in $\mathcal{X}_{g^{\prime} f}$ where $B_{n}$ is scalar multiplication by $\lambda_{n}$ on a subspace $P_{n}$ whose complement has finite relative dimension. Since $\left|\lambda_{n}\right| \leqq\left\|B_{n}\right\|$ and $\left\{\left\|B_{n}\right\|\right\}$ is a bounded set, $\left\{\lambda_{n}\right\}$ has at least one limit point. Let $\lambda$ be such a limit point. We assert that $\lambda$ is in the spectrum of $U H U^{-1} H^{-1}$. It will suffice to show that given $\epsilon>0$ there exists a unit vector $x$ such that $\left\|\left(U H U^{-1} H^{-1}-\lambda I\right) x\right\|<\epsilon$. To this end, choose $n$ so that

$$
\left\|U H U^{-1} H^{-1}-B_{n}\right\|<\epsilon / 2,\left|\lambda_{n}-\lambda\right|<\epsilon / 2,
$$


and choose $x$ a unit vector in $P_{n}$. Then

$$
\begin{gathered}
\left\|\left(U H U^{-1} H^{-1}-\lambda I\right) x\right\| \leqq \\
\left\|\left(U H U^{-1} H^{-1}-B_{n}\right) x\right\|+\left\|\left(B_{n}-\lambda_{n} I\right) x\right\| \\
+\left\|\left(\lambda_{n}-\lambda\right) I x\right\|<\epsilon / 2+\epsilon / 2=\epsilon .
\end{gathered}
$$

Now the product $T S$ of two positive operators one of which is invertible, say $T$, has real, non-negative spectrum, for such a product is conjugate to a positive operator,

$$
T S=T^{1 / 2}\left(T^{1 / 2} S^{1 / 2}\right)\left(T^{1 / 2} S^{1 / 2}\right)^{*} T^{-1 / 2} .
$$

Thus $\lambda$ is a positive number (nonzero since $U H U^{-1} H^{-1}$ is invertible).

Let $k$ be a (large) positive integer, and let a positive $\delta$ be assigned. Choose $n$ so that

$$
\left|\lambda_{n}^{k}-\lambda^{k}\right|<\delta /\left(2\left\|H^{-1}\right\|\right)
$$

and

$$
\left\|U H U^{-1} H^{-1}-B_{n}\right\|<\delta^{\prime}
$$

where

$$
\delta^{\prime}=\delta /\left(2 k\left\|H^{-1}\right\| \lambda^{\prime}\right)
$$

and $\lambda^{\prime}>\max \left\{1,\left|\lambda_{n}\right|, \cdots,\left|\lambda_{n}\right|^{k}\right\}$ (clearly, $\lambda^{\prime}$ need not depend on the $B_{n}$ chosen for if $\lambda_{n}$ is suitably near $\lambda$ we may choose $\lambda^{\prime}$ to be $\left.\max \left\{2,2 \lambda, \cdots, 2 \lambda^{k}\right\}\right)$. For $y$ a unit vector in $P_{n}$, we then have

$$
\left\|U H U^{-1} H^{-1} y-\lambda_{n} y\right\|<\delta^{\prime}
$$

so that

$$
\begin{aligned}
\| H^{-1} U^{-1}\left(U H U^{-1} H^{-1} y\right. & \left.-\lambda_{n} y\right) \| \\
& =\left\|U^{-1} H^{-1} y-\lambda_{n} H^{-1} U^{-1} y\right\| \leqq \delta^{\prime}\left\|H^{-1}\right\| .
\end{aligned}
$$

Now the spaces $U^{i}\left(P_{n}\right), i=0, \cdots, k-1$, each have a finite-dimensional complement so that the complement of their intersection, being a finite union of finite subspaces, is finite. Thus $\bigcap_{i=0}^{k-1} U^{i}\left(P_{n}\right)$ is infinite. Let $x$ be a unit vector in this intersection. Then

$$
\begin{aligned}
& \left\|U^{-k} H^{-1} x-\lambda_{n}^{k} H^{-1} U^{-k} x\right\| \\
& \leqq \\
& \leqq \sum_{i=1}^{k}\left\|\lambda_{n}^{k-i} U^{-i} H^{-1} U^{-k+i} x-\lambda_{n}^{k-i+1} U^{-i+1} H^{-1} U^{-k+i-1} x\right\| \\
& \quad=\sum_{i=1}^{b}\left|\lambda_{n}^{k-i}\right|\left\|U^{-1} H^{-1} U^{-k+i} x-\lambda_{n} H^{-1} U^{-1} U^{-k+i} x\right\|<\sum_{i=1}^{k}\left|\lambda_{n}^{k-i}\right| \delta^{\prime}\left\|H^{-1}\right\|,
\end{aligned}
$$

from $\left({ }^{*}\right)$, since $U^{-k+i} x$ is a unit vector in $P_{n}$, for $i=1, \cdots, k$. But then 
by choice of $\delta^{\prime}$. Moreover,

$$
\left\|U^{-k} H^{-1} x-\lambda_{n}^{k} H^{-1} U^{-k} x\right\|<\sum_{i=1}^{k}\left|\lambda_{n}^{k-i}\right| \delta^{\prime}\left\|H^{-1}\right\|<\delta / 2
$$

$$
\begin{aligned}
\left\|U^{-k} H^{-1} x-\lambda^{k} H^{-1} U^{-k} x\right\| & \leqq\left\|U^{-k} H^{-1} x-\lambda_{n}^{k} H^{-1} U^{-k} x\right\|+\left\|\left(\lambda_{n}^{k}-\lambda^{k}\right) H^{-1} U^{-k} x\right\| \\
& <\delta / 2+\left|\lambda_{n}^{k}-\lambda^{k}\right|\left\|H^{-1}\right\|<\delta .
\end{aligned}
$$

It follows that

$$
\lambda^{k}\left\|H^{-1} U^{-k} x\right\|<\delta+\left\|U^{-k} H^{-1} x\right\|=\delta+\left\|H^{-1} x\right\|
$$

and that

$$
\left\|H^{-1} x\right\|=\left\|U^{-k} H^{-1} x\right\|<\delta+\lambda^{k}\left\|H^{-1} U^{-k} x\right\|,
$$

where, it should be noted, $x$ depends on $\delta$ and $k$. However, since $U^{-k} x$ is a unit vector,

$$
\|H\|^{-1} \leqq\left\|H^{-1} U^{-k} x\right\|,
$$

from the relation

$$
\left.\left\|T^{-1}\right\|\right|^{-1}=\inf \{\|T z\|:\|z\|=1\}
$$

for $T$ a bounded operator (where $\left\|T^{-1}\right\|^{-1}$ has its natural meaning, 0 , when $T^{-1}$ does not exist as a bounded operator). Thus, combining this with the first inequality,

$$
\lambda^{k}\|H\|^{-1}<\delta+\left\|H^{-1} x\right\| \leqq \delta+\left\|H^{-1}\right\|,
$$

and this for arbitrary positive $\delta$ independent of $k$. Fixing $k$ and letting $\delta$ tend to zero, we have

$$
\lambda^{k}\|H\|^{-1} \leqq\left\|H^{-1}\right\|,
$$

for arbitrary positive integral $k$. This, of course, insures that $\lambda$ does not exceed 1. Employing the above noted relation again, we have

$$
\|B\|\left\|^{-1} \leqq\right\| H^{-1} x\left\|<\delta+\lambda^{k}\right\| H^{-1} U^{-k} x\left\|\leqq \delta+\lambda^{k}\right\| H^{-1} \|,
$$

with $\delta$ and $k$ independent. Fixing $k$ and letting $\delta$ tend to zero, we have

$$
\|H\|^{-1} \leqq \lambda^{k}\left\|H^{-1}\right\|,
$$

for arbitrary positive integral $k$, so that $\lambda$ is not less than 1 . Thus $\lambda$, an arbitrary limit point of the bounded sequence $\left(\lambda_{k}\right)$, is 1 , so that the sequence $\left(\lambda_{k}\right)$ has a limit and this limit is 1 . Suppose now that we replace each of the operators $B_{n}$ by the operators $B_{n}^{\prime}$ with $B_{n}^{\prime}$ scalar multiplication by 1 on $P_{n}$ and equal to $B_{n}$ on $I-P_{n}$. Clearly

$$
\left\|B_{n}-B_{n}^{\prime}\right\| \leqq\left|\lambda_{n}-1\right| \rightarrow 0,
$$


so that $U H U^{-1} H^{-1}$ is the limit of $\left(B_{n}^{\prime}\right)$. Thus $U H U^{-1} H^{-1}$ lies in $\mathscr{X}^{\prime} G_{f^{\prime}(1)}$ for arbitrary $A=U H$ in $G$. Since $U^{-1} H^{-1}$ is in $G$ as well as $U H$, we may assert that $U^{-1} H^{-1} U H$ is in $\mathscr{X}_{G_{f}(1)}$ as well as $U H U^{-1} H^{-1}$.

We have shown, in effect, that there exist subspaces with finite complements on which $U H$ and $H U$ agree to within any preassigned degree of accuracy. It was this to which we referred when we asserted that operators in $G$ have "large" portions which are "approximately normal."

The next theorem describes the situation with respect to the closed normal subgroups of the general linear group of a factor of type $I_{\infty}$.

THEOREM 4. If $\mathcal{X}$ is a factor of type $\mathrm{I}_{\infty}$ and $G$ is a closed, noncentral normal subgroup of $\mathcal{X}_{a}$, the general linear group of $\mathcal{X}$, then $G$ is the direct sum of $\mathcal{H}_{o_{f}(1)}$ and some closed subgroup of the scalars.

Proof. When we have completed the proof, it will follow from the theorem that $G$ is contained in $\mathcal{H}_{g j}$. We must establish this first, however. Suppose, then, that $G$ contains an operator $A$, with polar decomposition $U H$, which does not lie in $\mathcal{X}_{\theta f}$. If either of $U$ or $H$ were in $\mathcal{X}_{\theta_{f}}$, a nonzero scalar multiple of the other would lie in $G$. This scalar multiple, being normal, would, by Lemma 6 , lie in $\mathcal{X}_{g}$ so that $A$ would lie in $\mathscr{X}_{g_{f}}$ contrary to our assumption. We may assume, therefore, that both $U$ and $H$ have more than one center of infinite density. In particular, $H$ has at least two such centers $\alpha, \beta$. Suppose we can approximate to the set of operators $\left\{\alpha^{-1} \beta \xi P_{0}+\beta^{-1} \alpha \mu\left(I-P_{0}\right): P_{0}\right.$ equivalent to $\left.I-P_{0},|\xi|=|\mu|=1\right\}$ as closely as we wish with operators in $G$. This set of operators is compact (being the continuous image of a 2-torus) so that $G$ contains one of the operators in the set. Since $\alpha, \beta$ are positive and unequal, $\alpha^{2} \neq \beta^{2}$, so that

$$
0<\left|\alpha^{-1} \beta-\beta^{-1} \alpha\right| \leqq\left|\alpha^{-1} \beta \xi-\beta^{-1} \alpha \mu\right|
$$

for all $\xi, \mu$ such that $|\xi|=|\mu|=1$. Thus the given set of operators contains no scalars, and $G$ contains a noncentral operator of the form $\rho P_{0}+\tau\left(I-P_{0}\right)$. It follows, from Lemma 5 , that $G$ is all of $\mathcal{X}_{g}$, so that, if $G$ is to be proper, $G$ is contained in $\mathscr{X}_{g f}$.

Given $\epsilon>0$, it remains to approximate an operator of the given set (or a unitary transform of such an operator) to within $\epsilon$, by an operator in $G$. To this end, let $a, a^{\prime}$ be intervals containing $\alpha$ in their interior, $a$ open, $a^{\prime}$ closed, $a$ at positive distance from $\beta, a^{\prime}$ contained in $a$, and the length of $a$ less than $\tau$, where $\tau$ is a small positive number depending upon $\epsilon,\|H\|,\left\|H^{-1}\right\|, \alpha, \beta$; to be specified later. Let $E, E^{\prime}$ be the spectral projections for $H$ corresponding to $a, a^{\prime}$, respectively. Let $F, F^{\prime}, b, b^{\prime}$ be in the same situation with respect to the center $\beta$ for $H$, taking care that $E F=0$. Let $P_{1}, \cdots, P_{k}$ be spectral projections for $U$ corresponding to disjoint open or closed arcs which cover the unit circle, each arc of length less than $\tau$. Thus $P_{i} P_{j}=0$ and $\sum P_{t}=I$. Suppose that for none of the $P_{t}$ can one find an infinite subspace such that $E^{\prime}$ 
acting on this infinite subspace has a bounded inverse (i.e., $\left\|E^{\prime} x\right\|$ stays above a fixed positive constant as $x$ ranges over the unit vectors in the subspace). In this case, each infinite subspace of $P_{t}$, disjoint from $E^{\prime}$, contains a unit vector $x$ such that $\left\|E^{\prime} x\right\|$ is small. Each such subspace is therefore asymptotic (cf. $[1, \S 4,5,6]$ for terminology and results) to $I-E^{\prime}$, and $P_{t}$ is, accordingly, completely asymptotic to $I-E^{\prime}$. If $T$ is an arbitrary subspace, we define

$$
d\left(T, I-E^{\prime}\right)=\sup \left\{\left\|E^{\prime} x\right\|: x \text { in } T,\|x\|=1\right\} .
$$

We assert that given $\delta>0$ there exists a subspace $P_{t}^{\prime}$ of $P_{t}$ such that $P_{t}-P_{t}^{\prime}$ is finite and $d\left(P_{t}^{\prime}, I-E^{\prime}\right) \leqq \delta$. Indeed, if $P_{t}$ is finite choose $P_{t}^{\prime}=(0)$, otherwise suppose that each $P_{t}^{\prime}$ satisfying $P_{t}^{\prime} \subset P_{t}$, and $P_{t}-P_{t}^{\prime}$ is finite, is such that $d\left(P_{t}^{\prime}, I-E^{\prime}\right)>\delta$. Then, in particular, since $P_{t}$ itself satisfies these conditions, $d\left(P_{t}, I-E^{\prime}\right)>\delta$ and we can choose a unit vector $x_{1}$ in $P_{t}$ such that $\left\|E^{\prime} x_{1}\right\|>\delta$. Suppose $x_{1}, \cdots, x_{n-1}$ have been chosen in $P_{t}$ subject to the conditions that they are mutually orthogonal, each have norm 1 , and, for each $x_{i},\left\|E^{\prime} x_{i}\right\|>\delta$. Then, denoting by $\left[x_{1}, \cdots, x_{n-1}\right]$ the subspace generated by $x_{1}, \cdots, x_{n-1}$, $P_{t}-\left[x_{1}, \cdots, x_{n-1}\right]$ has the finite complement $\left[x_{1}, \cdots, x_{n-1}\right]$ in $P_{t}$ and, therefore, contains a unit vector $x_{n}$ such that $\left\|E^{\prime} x_{n}\right\|>\delta$. In this fashion, we construct an infinite orthonormal sequence $\left(x_{i}\right)$ in $P_{t}$ such that $\left\|E^{\prime} x_{i}\right\|>\delta$. But this is impossible since $\left(x_{i}\right)$ tends weakly to 0 and, in the notation of $[1$, p. 10$], \alpha\left(x_{i}, I-E^{\prime}\right)$ does not tend to 0 , contradicting $[1$, Théorème 1,5$]$. Then with $\delta<1 / k$ choose $P_{t}^{\prime}$ in $P_{t}$ so that $P_{t}-P_{t}^{\prime}$ is finite and $d\left(P_{t}^{\prime}, I-E^{\prime}\right)$ $<\delta$. Then

$$
I-\sum_{t=1}^{k} P_{t}^{\prime}=\sum_{t=1}^{k} P_{t}-\sum_{t=1}^{k} P_{t}^{\prime}=\sum_{t=1}^{k} P_{t}-P_{t}^{\prime}
$$

is finite. We assert that $E^{\prime}$ and $\sum_{t} P_{t}^{\prime}$ have a nonzero intersection. Suppose for the moment that this is so, and let $x$ be a unit vector in this intersection. Say $x=x_{1}+\cdots+x_{k}$ with $x_{t}$ in $P_{t}^{\prime}$. Then

$$
\begin{aligned}
1=\left\|E^{\prime} x\right\| & =\left\|E^{\prime} x_{1}+\cdots+E^{\prime} x_{k}\right\| \leqq\left\|E^{\prime} x_{1}\right\|+\cdots+\left\|E^{\prime} x_{k}\right\| \\
& \leqq\left\|x_{1}\right\| d\left(P_{1}^{\prime}, I-E^{\prime}\right)+\cdots+\left\|x_{k}\right\| d\left(P_{k}^{\prime}, I-E^{\prime}\right) \leqq k \delta<1 .
\end{aligned}
$$

This contradiction would show that $E^{\prime}$ acting on some infinite subspace of some $P_{t}$ has a bounded inverse. Call this $P_{t}, P^{\prime}$ and the infinite subspace on which $E^{\prime}$ has a bounded inverse $P^{\prime \prime}$. It remains, however, to show that the intersection of $\sum P_{t}^{\prime}$ and $E^{\prime}$ is not $(0)$. We shall show that if $P$ is a projection with finite complement and $Q$ is infinite (both in the same factor), then $P \cap Q$ is infinite. In fact, in [4, Lemma 7] it is proved (as is our case) that if $D(Q)>D(I-P)$, then $P \cap Q$ is nonzero. A glance at the proof of that lemma shows that what is actually proved is that

$$
D(P \cap Q)>D\left(Q_{1}\right)-D(I-P)
$$

where $Q_{1}$ is an arbitrary finite projection in $Q$. Consequently, $Q$ being in- 
finite and $I-P$ finite, $P \cap Q$ is infinite.

We apply the foregoing, now, with $F^{\prime}$ in place of $E^{\prime}$ and conclude that there exists a spectral projection $Q^{\prime}$ for $U$ corresponding to an arc of length less than $\tau$ which contains an infinite subspace $Q^{\prime \prime}$ on which $F^{\prime}$ has a bounded inverse. Say $\left\|E^{\prime} x\right\| \geqq \epsilon^{\prime}>0$ for $x$ a unit vector in $P^{\prime \prime}$ and $\left\|F^{\prime} y\right\| \geqq \epsilon^{\prime \prime}>0$ for $y$ a unit vector in $Q^{\prime \prime}$. Let the arcs to which $P^{\prime}, Q^{\prime}$ correspond be $c^{\prime}, d^{\prime}$, respectively, and let $c, d$ with corresponding spectral projections $P, Q$, respectively, be open arcs of length less than $\tau$ containing the closures of $c^{\prime}, d^{\prime}$, respectively. Let $\zeta, \eta$ be interior to $c^{\prime}, d^{\prime}$ respectively, and let $f, g, h, k$ be continuous, real, non-negative-valued functions which are 1 on $a^{\prime}, 0$ outside $a ; 1$ on $b^{\prime}, 0$ outside $b ; 1$ on the closure of $c^{\prime}, 0$ outside $c$; and 1 on the closure of $d^{\prime}$, 0 outside $d$, respectively. The spectral theory tells us that

$$
(I-E) f(H)=(I-F) g(H)=(I-P) h(U)=(I-Q) k(U)=0
$$

and

$$
E^{\prime} f(H)=E^{\prime}, \quad F^{\prime} g(H)=F^{\prime}, \quad P^{\prime} h(U)=P^{\prime}, \quad Q^{\prime} k(U)=Q^{\prime} .
$$

We now construct a subspace $R$ with finite complement such that for $x$ a unit vector in $R$

$$
\|f(H) h(U) x-h(U) f(H) x\|<\sigma
$$

and

$$
\|g(H) k(U) x-k(U) g(H) x\|<\sigma,
$$

where $\sigma$ is some small positive constant depending upon $\epsilon, \epsilon^{\prime}, \epsilon^{\prime \prime},\|H\|,\left\|H^{-1}\right\|$, $\alpha$ and $\beta$; to be specified later. It will suffice, of course, to perform the construction so that the first inequality holds on the constructed subspace; for, by symmetry, we can find a subspace on which the second inequality holds and then take for $R$ the intersection of the two spaces so constructed. Choose $p$ and $q$, polynomials of degree $m, n$, respectively, so as to approximate $f, h$, respectively, uniformly to within $\delta$ on intervals containing the spectra of $U$ and $H$. Since $U^{-1} H^{-1} U H$ and $H^{-1} U H U^{-1}$ are in $\mathscr{X}_{g_{f}(1)}$, we can find a subspace $R^{\prime}$ with finite complement such that

$$
\|U H x-H U x\|<\delta^{\prime},\left\|U^{-1} H x-H U^{-1} x\right\|<\delta^{\prime}
$$

for $x$ a unit vector in $R^{\prime}$. Let $R$ be the intersection of all the subspaces $T^{-1}\left(R^{\prime}\right)$, where $T$ runs through the monomials in $U, U^{*}, H$ of degree not exceeding $m$ in $H$ nor $n$ in $U, U^{*}$. Since $R$ is the intersection of a finite number of subspaces with finite complements, $R$ itself has a finite complement. Clearly, now, if $\delta^{\prime}$ is chosen sufficiently small depending upon $p, q$ and $\delta^{\prime \prime}$, then we have

$$
\left\|p(H) q\left(U, U^{*}\right) x-q\left(U, U^{*}\right) p(H) x\right\|<\delta^{\prime \prime}
$$


for $x$ a unit vector in $R$. Thus

$$
\|f(H) h(U) x-h(U) f(H) x\|<\sigma
$$

for $x$ a unit vector in $R$, if $\delta^{\prime \prime}$ and $\delta$ are chosen suitably small depending now upon $\sigma$ and the norms of $f(H), h(U)$.

We know that $P^{\prime \prime}, Q^{\prime \prime}$ are infinite, and that $R$ has a finite complement. Applying our previous remark to $P^{\prime \prime}, Q^{\prime \prime}$, and $R$, we have that $P^{\prime \prime} \cap R$, $Q^{\prime \prime} \cap R$ are infinite. It is easy to see that $f(H), g(H)$ have bounded inverses on $P^{\prime \prime} \cap R, Q^{\prime \prime} \cap R$, respectively. Indeed, if $x$ is a unit vector in $P^{\prime \prime} \cap R$,

$$
\begin{aligned}
\|f(H) x\|^{2} & =\left\|f(H) E^{\prime} x+f(H)\left(I-E^{\prime}\right) x\right\|^{2}=\left\|E^{\prime} x+\left(I-E^{\prime}\right) f(H) x\right\|^{2} \\
& =\left\|E^{\prime} x\right\|^{2}+\left\|\left(I-E^{\prime}\right) f(H) x\right\|^{2},
\end{aligned}
$$

and we know that $\left\|E^{\prime} x\right\|$ cannot be less than $\epsilon^{\prime}$. Thus $\epsilon^{\prime} \leqq\|f(H) x\|$, and, similarly, $\epsilon^{\prime \prime} \leqq\|g(H) y\|$ for all unit vectors $y$ in $Q^{\prime \prime} \cap R$. It follows from this that the ranges $M$ and $N$ of $f(H)\left(P^{\prime \prime} \cap R\right)$ and $g(H)\left(Q^{\prime \prime} \cap R\right)$, respectively, are closed and infinite. In fact, if $f(H) x_{n}$ has a limit with $\left(x_{n}\right)$ a sequence of vectors in $P^{\prime \prime} \cap R$, then $\left\|f(H) x_{n}-f(H) x_{m}\right\|$ tends to 0 as $n$ and $m$ tend, jointly, to $\infty$. But

$$
\left\|f(H)\left(x_{n}-x_{m}\right)\right\|=\left\|f(H)\left(x_{n}-x_{m}\right) /\right\| x_{n}-x_{m}\|\| \cdot\left\|x_{n}-x_{m}\right\| \geqq \epsilon^{\prime}\left\|x_{n}-x_{m}\right\|
$$

so that $x_{n}$ is a Cauchy sequence and has a limit $x$ in the closed subspace $P^{\prime \prime} \cap R$. Of course, $f(H) x$ is the limit of $\left(f(H) x_{n}\right)$, so that the ranges in question are closed. Let $T$ be the operator defined on $M$ as the inverse to $f(H)$ and on $I-M$ as 0 . The operator $T$ is uniquely characterized by the equations

$$
T M=T, \quad T f(H)\left(P^{\prime \prime} \cap R\right)=P^{\prime \prime} \cap R
$$

so that $T$ is in $\mathcal{X}$, for, with $U^{\prime}$ in $\mathcal{H}^{\prime}$

$$
\begin{aligned}
U^{\prime-1} T U^{\prime} M & =U^{\prime-1} T M U^{\prime}=U^{\prime-1} T U^{\prime}, \\
U^{\prime-1} T U^{\prime} f(H)\left(P^{\prime \prime} \cap R\right) & =U^{-1} T f(H)\left(P^{\prime \prime} \cap R\right) U^{\prime} \\
& =U^{\prime-1}\left(P^{\prime \prime} \cap R\right) U^{\prime}=P^{\prime \prime} \cap R .
\end{aligned}
$$

Thus $U^{\prime-1} T U^{\prime}=T$, and $T \in\left(\mathcal{X}^{\prime}\right)^{\prime}=\mathscr{X}$. Now $T M=T$ so that $T^{*}=M T^{*}$. Thus the closure of the range of $T^{*}$ is contained in $M$, and is, by [1, p. 142], equivalent to the closure of the range of $T$ (which is $P^{\prime \prime} \cap R$ ). Therefore, $M$ is infinite as is $N$. Let $V^{\prime}$ be a partial isometry from $M$ to $N$, and let $V$ be the unitary operator which is $V^{\prime}$ on $M, V^{\prime *}$ on $N$, and the identity on $I-M-N$. The operator $A^{-1} V^{-1} A V=A^{-1} V A V$ is in $G$, and

$$
\left\|A^{-1} V A V-\left(\alpha^{-1} \beta \zeta^{-1} \eta M+\alpha \beta^{-1} \zeta \eta^{-1} N+I-M-N\right)\right\|<3 \epsilon .
$$

In order to verify this inequality, we test it on unit vectors in the three orthogonal subspaces $M, N$, and $I-M-N$. First, for $x$ a unit vector in $M, x=f(H) z$ where $z$ is a vector in $P^{\prime \prime} \cap R$ of norm not exceeding $1 / \epsilon^{\prime}$. We have 


$$
\begin{aligned}
\left\|A^{-1} V A V x-\left(\alpha^{-1} \beta \zeta^{-1} \eta M+\alpha \beta^{-1} \zeta \eta^{-1} N+I-M-N\right) x\right\| \\
\quad=\left\|A^{-1} V A V x-\alpha^{-1} \beta \zeta^{-1} \eta x\right\|=\left\|A^{-1} V A(g(H) y)-\alpha^{-1} \beta \zeta^{-1} \eta x\right\|
\end{aligned}
$$

where $y$ is a vector in $Q^{\prime \prime} \cap R$ of norm not exceeding $1 / \epsilon^{\prime \prime}$, since $V x(=g(H) y)$ is a unit vector in $N$. Now:

$$
\begin{aligned}
\left\|A^{-1} V A g(H) y-\alpha^{-1} \beta \zeta^{-1} \eta x\right\| & \leqq\left\|A^{-1} V U(M g(H) y)-\beta A^{-1} V U g(H) y\right\| \\
& +\left\|\beta A^{-1} V U(g(H) k(U) y)-\beta A^{-1} V U(k(U) g(H) y)\right\| \\
& +\left\|\beta A^{-1} V(U k(U) g(H) y)-\beta \eta A^{-1} V(k(U) g(H) y)\right\| \\
& +\left\|\beta \eta A^{-1} V(k(U) g(H) y)-\beta \eta A^{-1} V(g(H) k(U) y)\right\| \\
& +\left\|\beta \eta H^{-1} U^{-1} f(H) z-\beta \eta H^{-1} U^{-1}(h(U) f(H) z)\right\| \\
& +\left\|\beta \eta H^{-1}\left(U^{-1} h(U) f(H) z\right)-\beta \zeta^{-1} \eta H^{-1}(h(U) f(H) z)\right\| \\
& +\left\|\beta \zeta^{-1} \eta H^{-1}(h(U) f(H) z)-\beta \zeta^{-1} \eta H^{-1}(f(H) h(U) z)\right\| \\
& +\left\|\beta \zeta^{-1} \eta\left(H^{-1} f(B) h(U) z\right)-\alpha^{-1} \beta \zeta^{-1} \eta x\right\|<\epsilon .
\end{aligned}
$$

Reading from left to right, on the right-hand side of the above inequality, we label the terms appearing there with Roman numerals corresponding to their position. We have

$$
\begin{aligned}
\mathrm{I} & \leqq\left\|A^{-1} V U\right\|\|H(g(H) y)-\beta g(H) y\|=\left\|H^{-1}\right\| \cdot\|H F g(H) y-\beta F g(H) y\| \\
& \leqq\left\|H^{-1}\right\| \cdot\|H F-\beta F\| \cdot\|g(H) y\| \leqq\left\|H^{-1}\right\| \tau, \\
\mathrm{II} & \leqq\left\|\beta A^{-1} V U\right\| \cdot \|(g(H) k(U)-k(U) g(H) y \| \\
& \leqq \beta\left\|H^{-1}\right\| \sigma\|y\| \leqq \beta\left\|H^{-1}\right\| \sigma\left(1 / \epsilon^{\prime \prime}\right), \\
\mathrm{III} & \leqq\left\|\beta A^{-1} V\right\| \cdot\|U Q k(U) g(H) y-\eta Q k(U) g(H) y\| \\
& \leqq \beta\left\|H^{-1}\right\| \cdot\|U Q-\eta Q\| \cdot\|k(U) g(H) y\| \leqq 2 \beta\left\|H^{-1}\right\| \tau, \\
\mathrm{IV} & \leqq\left\|\beta \eta A^{-1} V\right\| \cdot\|(k(U) g(H)-g(H) k(U)) y\| \\
& \leqq \beta\left\|H^{-1}\right\| \sigma\|y\| \leqq \beta\left\|H^{-1}\right\| \sigma\left(1 / \epsilon^{\prime \prime}\right), \\
\mathrm{V} & \leqq\left\|\beta \eta H^{-1} U^{-1}\right\| \cdot\|f(H) z-h(U) f(H) z\| \\
& =\beta\left\|H^{-1}\right\| \cdot\|f(H) h(U) z-h(U) f(H) z\| \leqq \beta\left\|H^{-1}\right\| \sigma\|z\| \leqq \beta\left\|H^{-1}\right\| \sigma\left(1 / \epsilon^{\prime}\right), \\
\mathrm{VI} & \leqq\left\|\beta \eta H^{-1}\right\| \cdot\left\|U^{-1} h(U) f(H) z-\zeta^{-1} h(U) f(H) z\right\| \\
& =\beta\left\|H^{-1}\right\| \cdot\left\|\left(U^{-1} P-\zeta^{-1} P\right) h(U) f(H) z\right\|<\beta\left\|\Psi^{-1}\right\| \cdot\|h(U) f(B) z\| \tau \\
& \leqq 2 \beta\left\|H^{-1}\right\| \tau,
\end{aligned}
$$

$\mathrm{VII} \leqq\left\|\beta \zeta^{-1} \eta H^{-1}\right\| \cdot\|h(U) f(H) z-f(H) h(U) z\| \leqq \beta\left\|H^{-1}\right\| \sigma\|z\| \leqq \beta\left\|H^{-1}\right\| \sigma\left(1 / \epsilon^{\prime}\right)$, VIII $\leqq \beta\left\|H^{-1} f(H) h(U) z-\alpha^{-1} x\right\|=\beta\left\|H^{-1} E f(B) z-\alpha^{-1} E f(H) z\right\|$

$$
\leqq \beta\left\|H^{-1} E-\alpha^{-1} E\right\| \cdot\|f(H) z\| \leqq \beta \tau^{\prime} .
$$

Regarding I and II, note that 


$$
g(H) y=g(H) Q^{\prime} y=g(H) k(U) Q^{\prime} y=g(H) k(U) y .
$$

For IV and V, note that

$$
V(g(H) k(U) y)=V(g(H) y)=V(V x)=x=f(H) z .
$$

In $\mathrm{V}$ we use

$$
f(H) z=f(H) P^{\prime} z=f(H) h(U) P^{\prime} z=f(H) h(U) z .
$$

The $\tau^{\prime}$ appearing in VIII is the maximum of the lengths of the two intervals about $\alpha^{-1}, \beta^{-1}$ obtained by taking the inverse of each of the numbers in $a$ and $b$ respectively. Because of the continuity of inversion at nonzero numbers, $\tau^{\prime}$ can be made as small as we please by choosing $\tau$ suitably small, depending upon $\alpha$ and $\beta$.

By symmetry, we obtain the inequality $(* *)$ for $x$ a unit vector in $N$. Clearly we can realize the inequality $(* *)$ by making suitable choices of $\tau$ and $\sigma, \tau$ dependent upon $\epsilon,\|H\|,\left\|H^{-1}\right\|, \alpha, \beta$, and $\sigma$ dependent upon $\epsilon, \epsilon^{\prime}, \epsilon^{\prime \prime}$, $\|H\|,\left\|H^{-1}\right\|, \alpha, \beta$. However, we reserve the final choice of $\tau, \sigma$ until we have determined the conditions engendered by the satisfaction of $\left(^{* *}\right)$ for $x$ a unit vector in $I-M-N$.

Before checking $\left(^{* *}\right)$ on unit vectors in $I-M-N$, we need some additional information. In the following set of inequalities, we show, successively, that $A^{*}$ acts very much like a scalar on both $M$ and $N$ and, from this, that $A$ leaves $I-M-N$ "almost invariant." Suppose then that $y$ is a unit vector in $M$ with $y=f(H) z$, where $z$ is in $P^{\prime \prime} \cap R$ and $\|z\| \leqq 1 / \epsilon^{\prime}$ :

$$
\begin{aligned}
\left\|A^{*} y-\alpha \zeta^{-1} y\right\|= & \left\|B U^{-1} f(H) h(U) z-\alpha \zeta^{-1} y\right\| \\
\leqq & \left\|H U^{-1} f(H) h(U) z-H U^{-1} h(U) f(H) z\right\| \\
& +\left\|H U^{-1} h(U) f(H) z-\zeta^{-1} H h(U) f(H) z\right\| \\
& +\left\|\zeta^{-1} H h(U) f(H) z-\zeta^{-1} H f(H) h(U) z\right\| \\
& +\left\|\zeta^{-1} H f(H) z-\alpha \zeta^{-1} y\right\| \\
\leqq & \|H\| \cdot\|z\| \sigma+\|H\| \cdot\|h(U) f(H) z\| \tau+\|H\| \cdot\|z\| \sigma+\tau \\
\leqq & \left(2\|H\| \sigma / \epsilon^{\prime}\right)+\tau(2\|H\|+1) \leqq \epsilon^{2} /\left(16\left\|H^{-1}\right\| 2 \cdot\|H\|\right) .
\end{aligned}
$$

Thus, if $x$ is a unit vector in $I-M$,

$$
\begin{aligned}
\|(I-M) A x-A x\|^{2} & =\|M A x\|^{2}=(M A x, A x)=\left(A^{*} M A x, x\right) \\
& =\left(A^{*} M A x-\alpha \zeta^{-1} M A x, x\right)+\left(\alpha \zeta^{-1} M A x, x\right) \\
& =\left(A^{*} M A x-\alpha \zeta^{-1} M A x, x\right) \\
& \leqq\left\|A^{*} M A x-\alpha \zeta^{-1} M A x\right\| \cdot\|x\| \\
& \leqq\|M A x\| \epsilon^{2} /\left(16\left\|H^{-1}\right\| 2\|H\|\right) \\
& \leqq\|H\| \epsilon^{2} /\left(\left.16\left\|H^{-1}\right\|\right|^{2} \cdot\|H\|\right)=\epsilon^{2} / 16\left\|H^{-1}\right\|^{2}
\end{aligned}
$$


so that

$$
\|(I-M) A x-A x\| \leqq \epsilon / 4\left\|H^{-1}\right\| .
$$

Similarly, for $x$ a unit vector in $I-N$ :

$$
\|(I-N) A x-A x\| \leqq \epsilon / 4\left\|H^{-1}\right\|,
$$

and, thus, for $x$ a unit vector in $I-M-N(=(I-M)(I-N))$ :

$$
\begin{aligned}
\|(I-M-N) A x-A x\|= & \|(I-M)(I-N) A x-A x\| \\
\leqq & \|(I-M)[(I-N) A x-A x]\| \\
& +\|(I-M) A x-A x\| \leqq\|(I-N) A x-A x\| \\
& +\|(I-M) A x-A x\| \leqq \epsilon / 2\left\|H^{-1}\right\| .
\end{aligned}
$$

We are now prepared to check the inequality $\left({ }^{* *}\right)$ on the unit vector $x$ in $I-M-N$ :

$$
\begin{aligned}
\| A^{-1} V A V x- & \left(\alpha^{-1} \beta \zeta^{-1} \eta M+\alpha \beta^{-1} \zeta \eta^{-1} N+I-M-N\right) x \| \\
= & \left\|A^{-1} V A x-x\right\| \leqq\left\|A^{-1} V A x-A^{-1} V(I-M-N) A x\right\| \\
& +\left\|A^{-1}(I-M-N) A x-A^{-1}(A x)\right\| \leqq 2\left\|H^{-1}\right\| \epsilon / 2\left\|H^{-1}\right\|=\epsilon .
\end{aligned}
$$

All of the foregoing estimates may be achieved by suitable choices of $\tau$ and $\sigma$ dependent upon the quantities previously mentioned.

Let $B=\alpha^{-1} \beta \zeta^{-1} \eta M+\alpha \beta^{-1} \zeta \eta^{-1} N+I-M-N$, and let $\alpha^{-1} \beta$ be that one of $\alpha^{-1} \beta, \alpha \beta^{-1}$ which does not exceed 1. Suppose, first, that $I-M-N$ is finite. The operator $T=\alpha^{-1} \beta \zeta^{-1} \eta(I-M-N)+M+N$ has norm 1 and lies in $\mathcal{X}_{a_{f}(1)}$ and hence in $G$. Thus $T A^{-1} V A V$ lies in $G$,

$$
T B=\alpha^{-1} \beta \zeta^{-1} \eta(I-N)+\alpha \beta^{-1} \zeta \eta^{-1} N,
$$

and

$$
\left\|T A^{-1} V A V-T B\right\| \leqq\|T\| \cdot\left\|A^{-1} V A V-B\right\| \leqq 3 \epsilon .
$$

But $T B$ is a unitary transform of an operator in the set $\left\{\alpha^{-1} \beta \xi P_{0}\right.$ $\left.+\alpha \beta^{-1} \mu\left(I-P_{0}\right)\right\}$. We would, therefore, have the desired approximation, and the proof would be complete. We may assume that $I-M-N$ is infinite. In this case choose $P_{1}, P_{2}$ orthogonal infinite projections in $I-M-N$ with sum $I-M-N$. Let $W_{1}$ be a partial isometry from $M$ to $P_{1}$ and $W_{2}$ be a partial isometry from $N$ to $P_{2}$. Let $W$ be the unitary operator which is $W_{1}$ on $M$, $W_{1}^{*}$ on $P_{1}, W_{2}$ on $N$, and $W_{2}^{*}$ on $P_{2}$. We have then

$$
B W B W=\alpha^{-1} \beta \zeta^{-1} \eta\left(M+P_{1}\right)+\alpha \beta^{-1} \zeta \eta^{-1}\left(N+P_{2}\right)
$$

which is a unitary transform of an operator in the set to which we wish to approximate by operators in $G$. On the other hand it is clear that

$$
\left\|\left(A^{-1} V A V\right) W\left(A^{-1} V A V\right) W-B W B W\right\|
$$


is small (so long as $\epsilon$ is made sufficiently small, dependent upon $\alpha$ and $\beta$ ). Now $\left(A^{-1} V A V\right) W\left(A^{-1} V A V\right) W$ is in $G$ so that, in any event, we have our desired approximation and $G$ is contained in $\mathscr{x}_{g \rho}$.

Suppose, now, that $\mathcal{H}$ is a closed, normal subgroup of $\mathcal{X}_{g f}$. The same proof which showed that the $\mathcal{H C}$ of Lemma 6 , which was normal in $\mathcal{X}_{g f(1)}$, contains a noncentral unitary operator shows that the $\mathfrak{H C}$ of our present argument contains a noncentral unitary operator (with the minor modification that, once the noncentral normal operator $A$ is constructed in $\mathfrak{H}$, we must use its center of infinite density in those places of the proof of Lemma 6 where 1 was used as the center of infinite density for the $A$ of that proof). It follows from $[4$, Theorem 4$]$ that $\mathcal{H C}$ contains $G_{f}^{(1)}$, so that $\mathcal{H}$ contains $\mathcal{X}_{g f(1)}$, by Lemma 6. It follows, immediately, that $\mathfrak{F C}$ is the direct product of $\mathcal{X}_{g_{f}(1)}$ and the subgroup of the scalars in which it intersects the scalars. This scalar subgroup must, of course, be closed, since $\mathfrak{H C}$ is closed, and the proof is complete.

4. Conclusion. We state a corollary to the foregoing results, on approximation by invertible operators, which is completely analogous to Corollary 1 of [4].

CoROllary 1. Let $\mathcal{X}$ be a factor and $\mathrm{p}$ a property of invertible operators which is invariant under similarity (inner transform) and such that an operator has property $\mathrm{p}$ only if its inverse has property $\mathrm{p}$. If some noncentral, invertible operator $\mathrm{A}$ has property $\mathrm{p}$ ( $A$ not in $\mathcal{H}_{o f}$ in the $\mathrm{I}_{\infty}, \mathrm{II}_{\infty}$ cases, $A$ normal in the $\mathrm{II}_{\infty}$ case), then for each invertible operator $B$ in $M$ and each positive $\epsilon$ there exists a finite set $B_{1}, \cdots, B_{n}$ of invertible operators in $\mathcal{H}$, each with property $\mathrm{p}$, and some positive scalar $\alpha$ such that

$$
\left\|\alpha B-B_{1} \cdots B_{n}\right\|<3 \epsilon .
$$

If $\mathcal{X}$ is not of type $\mathrm{II}_{1}, \alpha$ may be taken as 1 .

Proof. Immediate, from the results of the preceding section.

Almost all the concluding remarks of [4] are applicable to the present situation. Again the purely algebraic question remains untouched. Does Theorem 1 hold without the topological assumption of uniform closedness on the normal subgroups considered? As topological results, those we present are the strongest possible. The extension of our results to the nonseparable case is a routine matter.

In connection with Theorem 1, several remarks are of interest. In the first place, the topological commutator subgroup of $\mathcal{X}_{g}$, the general linear group of a factor of type $\mathrm{II}_{1}$, coincides with $\mathcal{X}_{g_{1}}$, the determinant 1 subgroup of $\mathcal{X}_{g}$. Indeed, the topological commutator subgroup of $\mathscr{X}_{\sigma}$ is a closed, normal subgroup of $\mathcal{X}_{g}$ and so contains $\mathscr{X}_{g_{1}}$, according to Theorem 1. On the other hand, each operator in the topological commutator subgroup has determinant 1 , from which our assertion follows. Another immediate consequence of Theorem 1 is the fact that each character of $\mathcal{X}_{\theta}, \mathcal{X}$ of type $\mathrm{II}_{1}$, which is continuous 
in the uniform topology is the composition of the determinant map and a character of the positive reals. This follows at once from the fact that the kernel of the given character contains $\mathcal{X}_{\sigma_{1}}$.

Finally, with regard to the lack of complete information in the $\mathrm{II}_{\infty}$ case, it should be observed that the proof of Theorem 4 fails for the $\mathrm{II}_{\infty}$ situation at only one point. We require some results of infinite-dimensional geometry to establish the existence of an infinite-dimensional subspace $F^{\prime}$ of a spectral projection $F$ for a unitary operator $U$ corresponding to an arc of the unit circle of length less than some preassigned positive number such that some other infinite projection $E$, with infinite complement, has a bounded inverse on $F^{\prime}$. All subspaces must lie in the given factor, of course. We establish the existence of such an $F^{\prime}$, in the $\mathrm{I}_{\infty}$ case, by means of the result: if $E$ is an infinite subspace completely asymptotic (cf. $[1$, p. 13]) to $F$, then $F$ contains a projection $F_{1}$ such that $F-F_{1}$ is finite and the angle between any vector in $E$ and the subspace $F_{1}$ is less than a preassigned $\delta(>0)$. It seems likely that this statement holds in the $\mathrm{II}_{\infty}$ case, with the obvious extension of "completely asymptotic" to this situation; and, with this result, Theorem 4 can be stated for both $\mathrm{I}_{\infty}$ and $\mathrm{II}_{\infty}$. The proof of this geometric result relative to a $\mathrm{II}_{\infty}$ requires an, as yet, undeveloped infinite-dimensional geometry relative to a $\mathrm{II}_{\infty}$.

\section{BIBLIOGRAPHY}

1. J. Dixmier, Etude sur les variêtés et les opérateurs de Julia, avec quelques applications Théses, Paris, 1949.

2. B. Fuglede and R. Kadison, On determinants and a property of the trace in finite factors, Proc. Nat. Acad. Sci. U.S.A. vol. 37 (1951) pp. 425-431.

3. - Determinant theory in finite factors, Ann. of Math. vol. 55 (1952) pp. 520-530. 399.

4. R. Kadison, Infinite unitary groups, Trans. Amer. Math. Soc. vol. 72 (1952) pp. 386-

5. F. Murray and J. von Neumann, Rings of operators, Ann. of Math. vol. 37 (1936) pp. 116-229.

Institute for Advanced Study, Princeton, N. J.

Columbia University, NEW YoRK, N. Y. 